\title{
Crocin prevents platelet-derived growth factor BB-induced vascular smooth muscle cells proliferation and phenotypic switch
}

\author{
LIJIAN TONG and GUOXIAN QI
}

Department of Geriatrics, The First Affiliated Hospital of China Medical University, Shenyang, Liaoning 110001, P.R. China

Received December 31, 2017; Accepted March 29, 2018

DOI: $10.3892 / \mathrm{mmr} .2018 .8854$

\begin{abstract}
The phenotypic switch of vascular smooth muscle cells (VSMCs) is a major initiating factor for atherosclerotic cardiovascular diseases. Platelet-derived growth factor-BB (PDGF-BB) initiates a number of biological processes that contribute to VSMC proliferation and phenotypic switch. Crocin, a component of saffron, has been reported to inhibit atheromatous plaque formation. However, the effects of crocin on PDGF-BB-induced VSMC proliferation and phenotypic switch remain unclear. The aim of the present study was to investigate the role of crocin on PDGF-BB-induced VSMCs proliferation and phenotypic switch and its underlying mechanisms. Cell proliferation and markers of VSMCs phenotypic switch were measured using a Cell Counting Kit-8 assay and western blot analysis, respectively. The signaling pathways involved in the effects of crocin on VSMCs were validated by western blot analysis with or without the use of specific pathway inhibitors. Crocin significantly inhibited PDGF-BB-induced VSMCs proliferation compared with the PDGF-BB only group $(\mathrm{P}<0.05)$. In addition, crocin significantly abrogated the PDGF-BB-induced increase in contractile protein $\alpha$-smooth muscle actin, calponin and decrease in synthetic proteins osteopontin (OPN) in a concentration dependent manner $(\mathrm{P}<0.05)$. In addition, crocin slowed PDGF-BB-induced Janus kinase (JAK)-signal transducer and activator of transcription 3 (STAT3) and extracellular signal-regulated kinase (ERK)/Kruppel-like factor 4 (KLF4) signaling activation in VSMCs. By applying the JAK inhibitor (AG490) and ERK1/2 inhibitor (U0126), the results suggested that the crocin
\end{abstract}

Correspondence to: Dr Guoxian Qi, Department of Geriatrics, The First Affiliated Hospital of China Medical University, 155 Nanjing North Street, Heping, Shenyang, Liaoning 110001, P.R. China E-mail: qgxian51a@163.com

Abbreviations: VSMCs, vascular smooth muscle cells; PDGF-BB, platelet-derived growth factor-BB; $\alpha$-SMA, $\alpha$-smooth muscle actin; OPN, osteopontin; STAT3, signal transducers and activators of transcription; ERK1/2, extracellular signal-regulated kinase 1/2; KLF4, kruppel-like factor 4

Key words: vascular smooth muscle cells, platelet-derived growth factor-BB, proliferation, phenotypic switch, crocin inhibited PDGF-BB-induced VSMCs phenotypic switch through the JAK/STAT3 and ERK/KLF4 signaling pathways. These results suggested that crocin may effectively prevent PDGF-BB-induced VSMCs proliferation and phenotypic switch and may be a promising candidate for the therapy of atherosclerotic cardiovascular diseases.

\section{Introduction}

Vascular smooth muscle cells (VSMCs) play an important role in blood vessel tone regulation and in vascular growth and response to injuries (1). In the media layer of mature blood vessels, VSMCs exhibit extensive plasticity and can go through phenotypic modulation from a quiescent contractile state to a proliferative synthetic state in response to various environmental stimuli, such as growth factors, reactive oxidative species, and mechanical injury (2). It is noteworthy that among abovementioned stimuli, platelet-derived growth factor-BB (PDGF-BB) modulated phenotypic switch has been thoroughly established and subsequently leads to the formation of neointima in response to vascular injury $(3,4)$. This phenotypic modulation is characterized by the alteration of proliferation and alterations in the expression of phenotypic markers, such as alpha smooth muscle actin ( $\alpha$-SMA), calponin and osteopontin (OPN) (5). Prevention of PDGF-BB-induced VSMC phenotypic switch and proliferation leading to the attenuated intimal hyperplasia and vascular remodeling (6). On this basis, deeper understanding of the mechanisms that may control VSMCs phenotype modulation could be a critical therapeutic target in treatment of atherosclerotic cardiovascular diseases.

Crocin is one of the major biologically active substances of saffron. The well-known pharmacological effects of crocin are anti-oxidant (7), anti-cancer (8) and neuroprotective activities (9). Previously, crocin was shown with the ability to significantly inhibit atheromatous plaque formation in atherosclerotic quails and its mechanisms may through decreasing the EC apoptosis and inhibiting the elevated calcium ion in oxidatively modified low-density lipoprotein induced VSMCs (10). Recently, a study found crocin could decrease blood lipid levels and inhibit lipogenesis by suppressing the expression of lipogenesis-related proteins and elevating lipid catabolism-related proteins (11). Moreover, crocin could alleviate the inflammation in a VD3-induced rat coronary atherosclerosis model by inhibiting $N F-\kappa B$ p65 nuclear 
translocation (11). These all suggested the potential protective effects of crocin in the initiation and progression of atherosclerosis. Nevertheless, to date, whether crocin could regulate VSMC phenotypic switch still needs to be elucidated.

In the present study, we aimed to explore the role of crocin in PDGF-BB-mediated phenotype switching of VSMCs. Primary rats aortic VSMCs were treated with PDGF-BB followed by various concentration of crocin. The proliferative rates and phenotypic switch of VSMCs were then measured. Subsequently, the potent mechanisms of the action were investigated.

\section{Materials and methods}

Cell and reagents. VSMCs were isolated from thoracic aortas of male SD rats as previous indicated (12). Animal studies conformed to the ARRIVE guidelines (2013) and were approved by the Institutional Animal Care and Use Committee of the First Hospital of China Medical University. The culture medium was Dulbecco's modified Eagle's media (DMEM; Gibco; Thermo Fisher Scientific, Inc., Waltham, MA, USA) with $100 \mathrm{U} / \mathrm{ml}$ penicillin/100 $\mu \mathrm{g} / \mathrm{ml}$ streptomycin (Invitrogen; Thermo Fisher Scientific, Inc.), containing 10\% fetal bovine serum (Gibco; Thermo Fisher Scientific, Inc.). Cells were maintained at $37^{\circ} \mathrm{C}$ and $5 \% \mathrm{CO}_{2}$. VSMCs of passages 5-9 were used for experiments. Crocin (cat. no. 42553-65-1, purity >97\%, structure presented in Fig. 1A) and PDGF-BB were purchased from Sigma-Aldrich (St. Louis, MO, USA). Antibodies against $\alpha$-SMA, OPN, calponin and kruppel-like factor 4 (KLF4) were obtained from Abcam (Cambridge, MA, USA). Antibodies against phosphorylated (p-)JAK1, JAK1, p-JAK2, JAK2, signal transducers and activators of transcription (p-STAT3), STAT3, extracellular signal-regulated kinase (p-ERK1/2), and ERK1/2 were purchased from Cell Signaling Technology, Inc. (Danvers, MA, USA). Antibodies against GAPDH was obtained from Santa Cruz Biotechnology, Inc. (Dallas, TX, USA). AG490 and U0126 were acquired from MedChem Express (Monmouth Junction, NJ, USA). The concentration of inhibitors was selected according to previous studies $(13,14)$.

Cell treatment. After an initial $24 \mathrm{~h}$ of culture in serum-free medium, VSMCs were exposed to $20 \mathrm{ng} / \mathrm{ml}$ PDGF-BB for $24 \mathrm{~h}$ with or without various concentration of crocin $(10,50$, $100 \mu \mathrm{M})$. For pathways inhibitors, VSMCs were pre-treatment for $1 \mathrm{~h}$ with AG490 or U0126 at the final concentration of 10 and $50 \mu \mathrm{M}$, respectively.

Cell viability assay. VSMCs were cultured in 96-well plates $\left(5 \times 10^{3}\right.$ cells/well). After reaching a confluence of $85 \%$, cells were treated with different concentration of crocin, and stimulated with $20 \mathrm{ng} / \mathrm{ml}$ PDGF-BB for $24 \mathrm{~h}$. Then, $10 \mu \mathrm{l}$ Cell Counting Kit-8 (CCK-8) reagent was added to each well followed by incubation for an additional $2 \mathrm{~h}$ at $37^{\circ} \mathrm{C}$. The absorbance of cells was measured at $450 \mathrm{~nm}$ using a microplate reader.

Immunofluorescence staining. Following treatment of VSMCs with PDGF and crocin, cells were fixed in $4 \%$ paraformaldehyde for $15 \mathrm{~min}$ and permeabilized in $0.1 \%$ Triton X-100 for another $15 \mathrm{~min}$ at room temperature. Subsequently, cells were blocked with $2 \%$ BSA for $30 \mathrm{~min}$ at room temperature followed by incubation with $\alpha$-SMA antibody (1:400) at $4^{\circ} \mathrm{C}$ overnight. After washes with PBS for three times, cells were incubated with goat anti-rabbit IgG H\&L Alexa Fluor $^{\circledR} 488$ (1:500) at room temperature for another $1 \mathrm{~h}$. Finally, nuclei were stained with DAPI for $5 \mathrm{~min}$. Images were taken under a fluorescence microscope (IX73-A12FL/PH; Olympus, Tokyo, Japan) at 200x magnification.

Western blot analysis. After treatment, the cells were lysed in RIPA lysis buffer supplemented with protease/phosphatase inhibitor for $15 \mathrm{~min}$ at $4^{\circ} \mathrm{C}$ and total protein concentrations were measured using the BCA assay. Protein samples were loaded on $10-12 \%$ SDS-PAGE and transferred to PVDF membranes followed by blocked with 5\% skim milk in TBST at room temperature for $1 \mathrm{~h}$. After washing, the membranes were incubated with the primary antibodies against $\alpha$-SMA $(1: 1,000)$, calponin $(1: 1,000)$, OPN (1:500), p-JAK1 $(1: 1,000)$, JAK1 (1:1,000), p-JAK2 (1:1,000), JAK2 (1:1,000), p-STAT3 $(1: 1,000)$, STAT3 $(1: 1,000)$, KLF4 $(1: 1,000)$, p-ERK1/2 $(1: 10,000)$, ERK $1 / 2(1: 10,000)$ and GAPDH $(1: 1,000)$ overnight at $4^{\circ} \mathrm{C}$. After three washes with TBST, the blots were incubated for $1 \mathrm{~h}$ at room temperature with HRP-conjugated goat anti-rabbit antibody/mouse antibodies at room temperature for $1 \mathrm{~h}$. Signals were visualized using an enhanced chemiluminescence kit and densitometry analysis of the immunoblots was carried out using Image J software (National Institute of Health, Bethesda, MD, USA).

Data analysis. All data are expressed as mean $\pm \mathrm{SD}$ and analyzed using SPSS version 19.0 (SPSS, Inc., Chicago, IL, USA). Statistical analyses were performed using one-way ANOVA with Tukey's post hoc test. The value of $\mathrm{P}<0.05$ was statistically significant.

\section{Results}

Crocin inhibits PDGF-BB-induced VSMCs proliferation and phenotypic switch. First, we assessed the effect of crocin on VSMCs proliferation. As shown in Fig. 1B, treatment with different concentrations $(10,50$, and $100 \mu \mathrm{M})$ of crocin for 24 or $48 \mathrm{~h}$ did not affect the proliferation of the VSMCs, as detected by CCK-8 assay. Then, we investigated the effects of crocin on PDGF-BB $(20 \mathrm{ng} / \mathrm{ml})$ treated VSMCs. The results in Fig. 2A showed that cell proliferation rate was dramatically increased in PDGF-BB treated VSMCs and was decreased with additional treatment with crocin in a concentration dependent manner. We hypothesized that the increased VSMCs proliferation rate may largely due to the transition from a contractile phenotype to a synthetic phenotype. Therefore, the markers of VSMCs phenotypic switch were tested under crocin treatment. Indicated by western blot analysis, PDGF-BB treatment significantly decreased the expression levels of $\alpha$-SMA and calponin, whereas increased the expression levels of OPN. Intriguing, these alterations were reversed by crocin in a concentration-dependent manner (Fig. 2B-E). Moreover, the results of immunofluorescent staining with $\alpha$-SMA revealed that compared with control group, PDGF-BB not only reduced the fluorescence intensity of $\alpha$-SMA, but also perturbed myofibrillar arrangement in the cytoplasm. After crocin treatment, above changes were gradually abolished (Fig. 2F). 
A

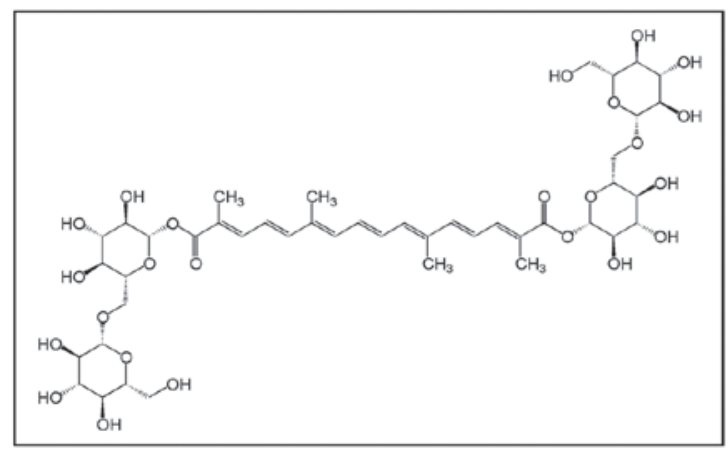

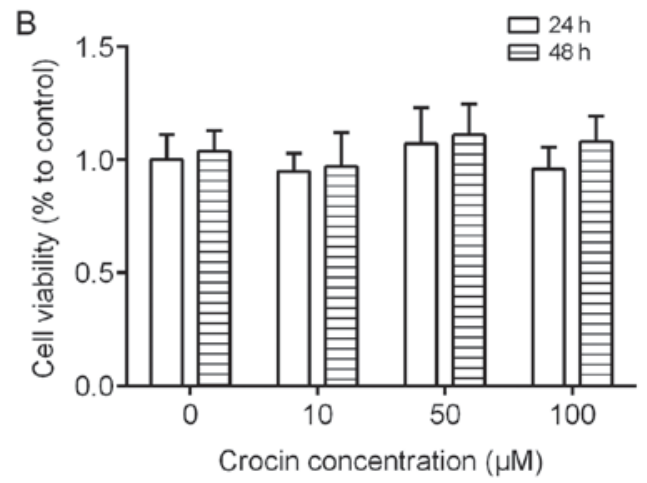

Figure 1. (A) Chemical structure of crocin. (B) Cell Counting Kit-8 assay was performed to determine vascular smooth muscle cells proliferation rate with the treatment of different concentration of crocin $(0,10,50$, and $100 \mu \mathrm{M})$ at 24 and $48 \mathrm{~h}$.

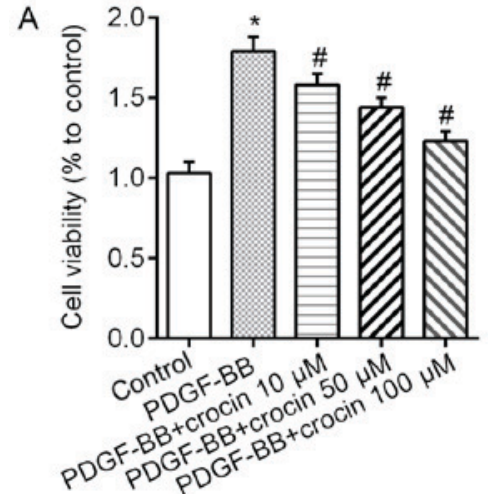

B

C

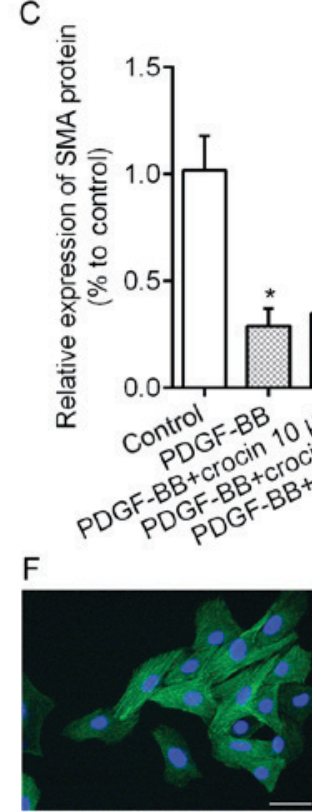

Control

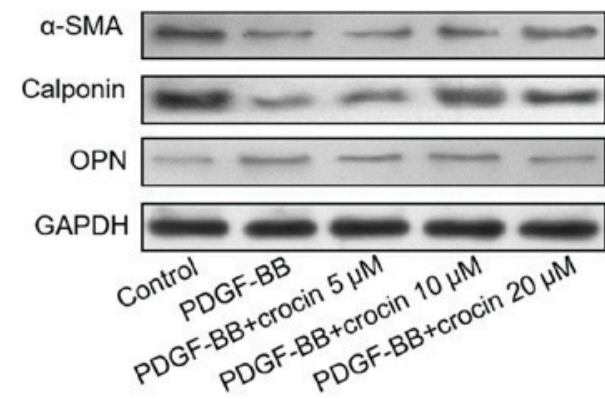

E

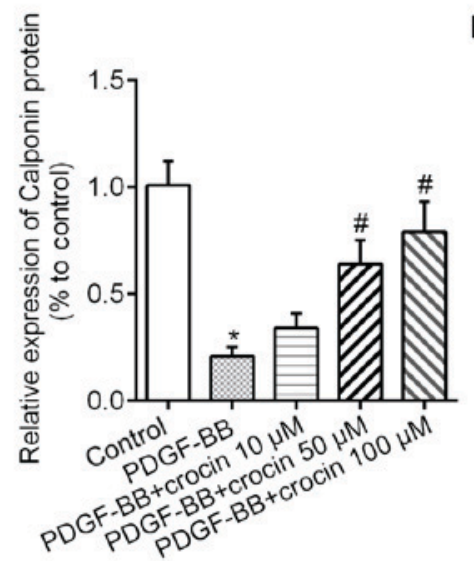

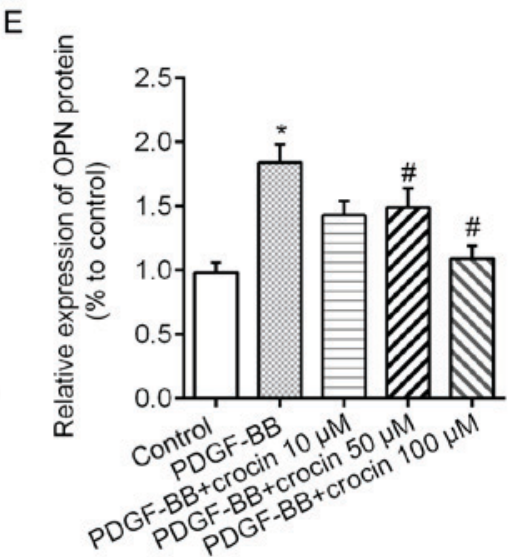

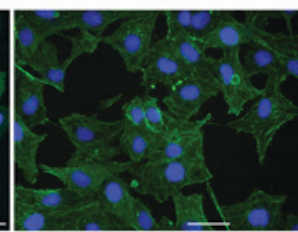

PDGF-BB

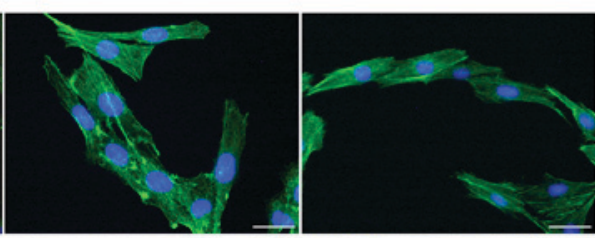

PDGF-BB+crocin $10 \mu \mathrm{M}$ PDGF-BB+crocin $50 \mu \mathrm{M}$ PDGF-BB+crocin $100 \mu \mathrm{M}$

Figure 2. Crocin abrogated PDGF-BB-induced VSMCs proliferation and phenotypic switch. (A) Cell Counting Kit-8 assay was used for the detection of VSMC proliferation rates in different groups. (B) Western blot analysis was conducted to quantify the expression levels of contractile protein $\alpha$-SMA, calponin and synthetic proteins OPN. Bar graph showing the relative protein levels of (C) $\alpha$-SMA, (D) calponin and (E) OPN. (F) Immunofluorescent staining of $\alpha$-SMA (green) of VSMCs in different groups. DAPI staining (blue) indicated the nucleus. Scale bar, $50 \mu \mathrm{m}$. ${ }^{*} \mathrm{P}<0.05$ vs. control; " $\mathrm{P}<0.05$ vs. PDGF-BB. OPN, osteopontin; SMA, smooth muscle actin; VSMC, vascular smooth muscle cell; PDGF-BB, platelet-derived growth factor-BB.

Crocin inhibits PDGF-BB-induced activation of JAK/STAT3 and ERK/KLF4 signaling pathways in VSMCs. We then examined the underlying mechanism of the inhibitory effect of crocin to VSMCs and JAK/STAT3 and ERK/KLF4 signaling pathways were investigated. Fig. 3A demonstrated that VSMCs exposure to PDGF-BB resulted in significant increases in the phosphorylation levels of JAK1, JAK2 and STAT3. Treatment of crocin dramatically downregulated PDGF-BB-induced 

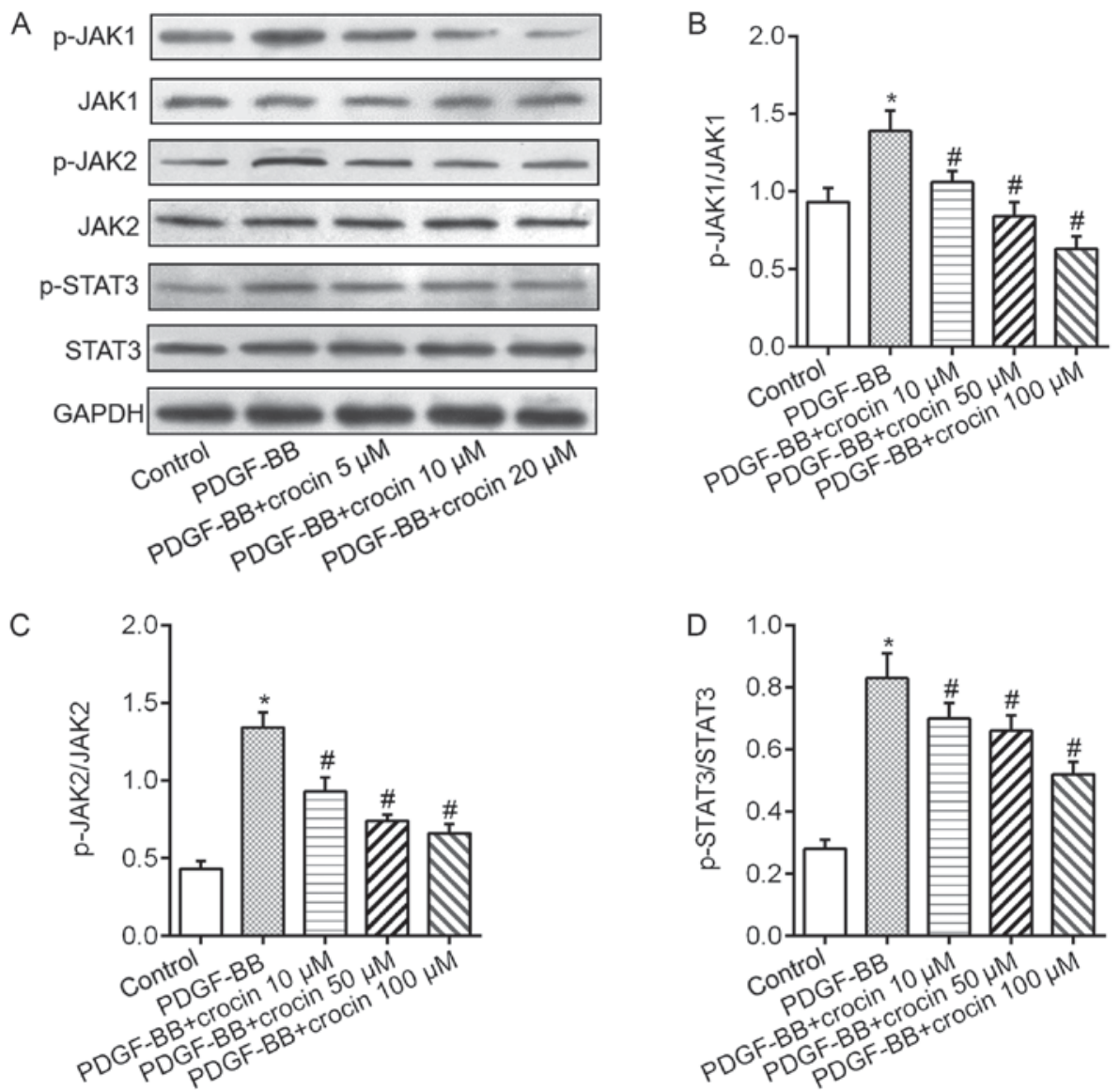

Figure 3. Crocin inhibited PDGF-BB-induced JAK/STAT3 signaling activation. (A) Representative images showing the protein expression of phosphorylated and total JAK1, JAK2 and STAT3. Bar graph showing the relative protein levels of phosphorylated (B) JAK1, (C) JAK2 and (D) STAT3. "P $<0.05$ vs. control; " $\mathrm{P}<0.05$ vs. PDGF-BB. PDGF-BB, platelet-derived growth factor-BB; STAT3, signal transducers and activators of transcription; JAK, Janus kinase; p-, phosphorylated.

A

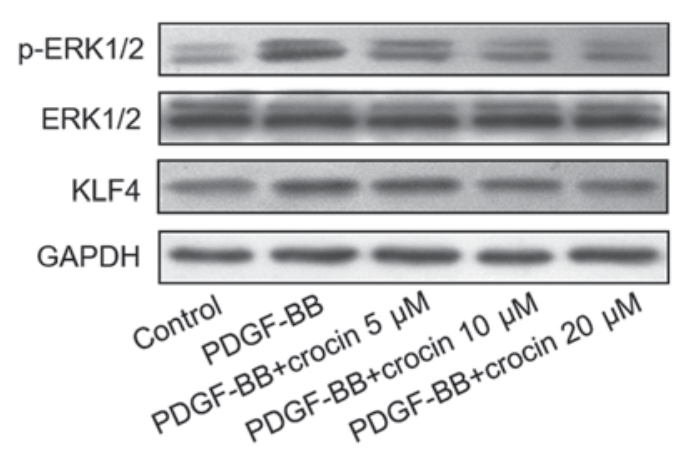

B

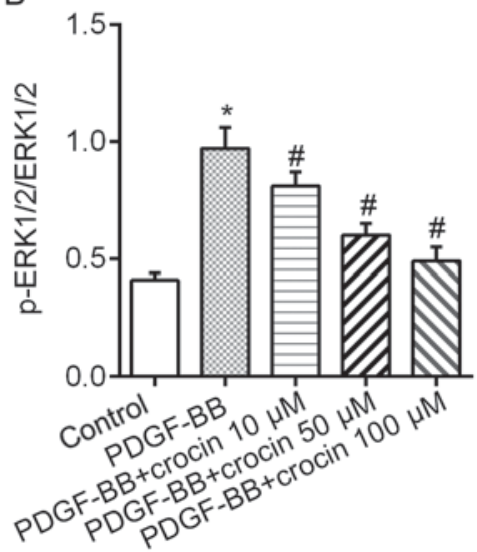

C

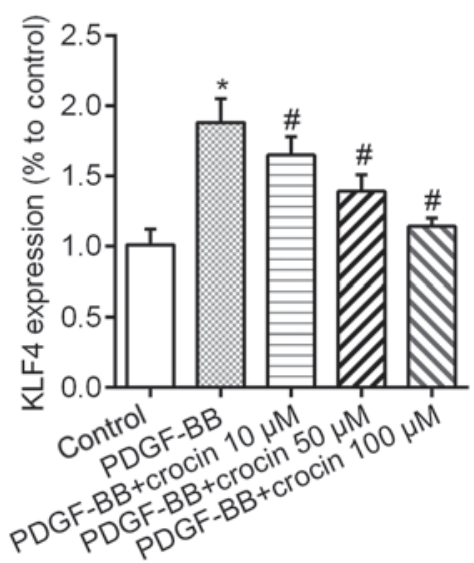

Figure 4. Crocin suppressed PDGF-BB-induced ERK/KLF4 signaling activation. (A) Representative images showing the protein expressions of phosphorylated and total ERK1/2 and expression levels of KLF4 protein. Bar graph showing the relative protein levels of (B) phosphorylated ERK1/2 and expression levels of (C) KLF4 in different groups. " $\mathrm{P}<0.05$ vs. control; ${ }^{~} \mathrm{P}<0.05$ vs. PDGF-BB. PDGF-BB, platelet-derived growth factor-BB; ERK, extracellular signal regulated kinase; KLF4, kruppel-like factor 4; p-, phosphorylated.

phosphorylation levels of JAK1, JAK2 and STAT3 in a concentration-dependent fashion (Fig. 3B-D). Additionally, in cultured VSMCs, exposure to PDGF-BB caused the elevated phosphorylation levels of ERK1/2 and increased expression levels of KLF4 protein (Fig. 4A). When the cells were co-treated with various concentration of crocin, above changes were abrogated in a concentration-dependent manner (Fig. 4B and C).
JAK/STAT3 and ERK/KLF4 signaling pathways mediate $P D G F-B B$-induced phenotype switching and VSMCs proliferation. We subsequently tested whether inhibition of the JAK/STAT3 and ERK/KLF4 signaling pathways were involved in PDGF-BB-induced phenotypic switching and proliferation of VSMCs. We found that administration of JAK inhibitor AG490 and ERK1/2 inhibitor U0126 significantly diminished 

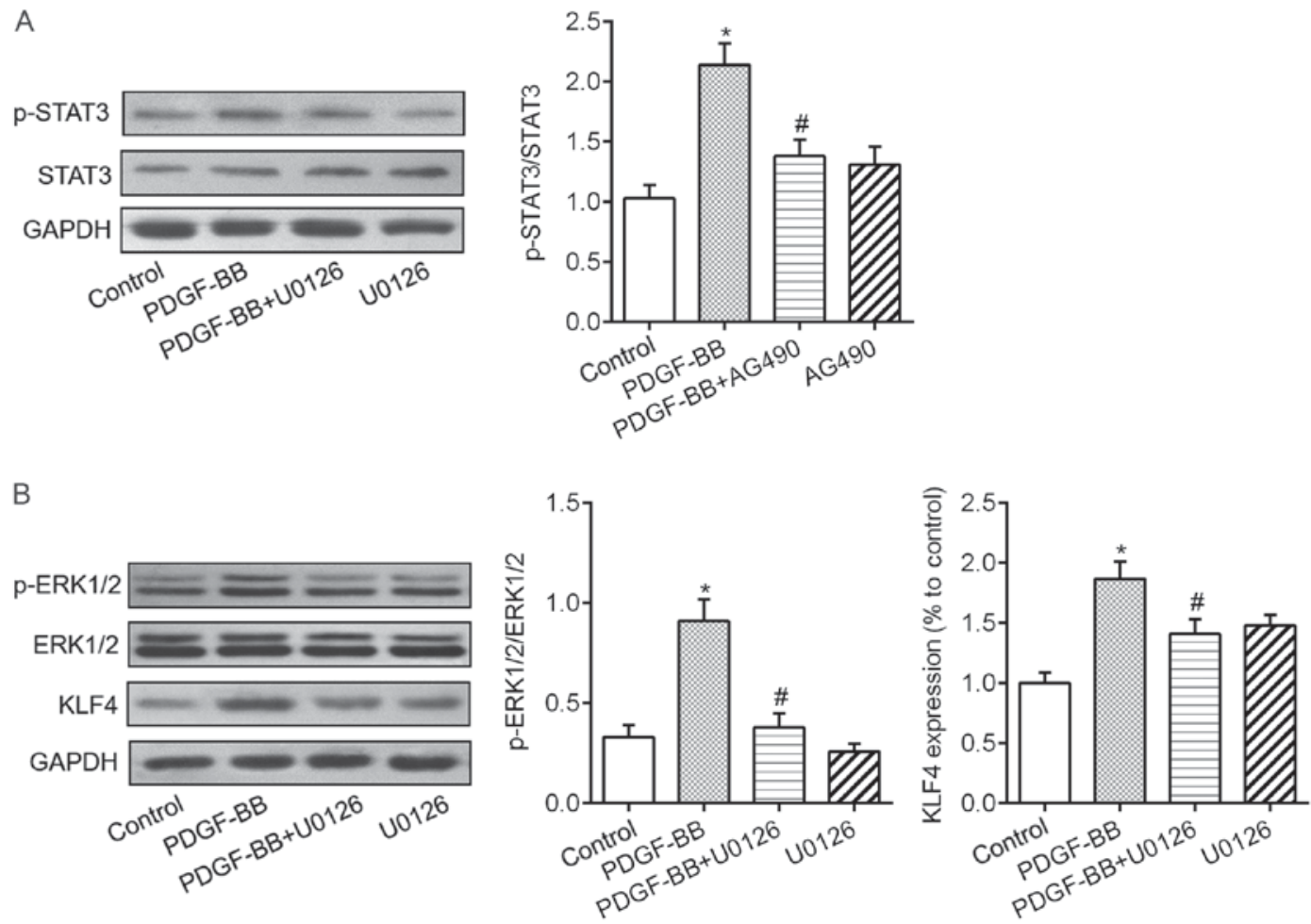

Figure 5. Suppression of PDGF-BB-induced JAK/STAT3 and ERK/KLF4 activation by AG490 and U0126 in VSMCs. (A) Representative western blot analysis showed phosphorylation of STAT3 in VSMCs treated with PDGF-BB $(20 \mathrm{ng} / \mathrm{ml})$ in the absence and presence of a specific JAK inhibitor AG490 (10 $\mu$ M). (B) Representative images showed phosphorylation of ERK1/2 and expression levels of KLF4 protein in VSMCs treated with PDGF-BB (20 ng/ml) in the absence and presence of ERK1/2 inhibitor U0126 $(50 \mu \mathrm{M}) .{ }^{~} \mathrm{P}<0.05$ vs. control; ${ }^{*} \mathrm{P}<0.05$ vs. PDGF-BB. PDGF-BB, platelet-derived growth factor-BB; ERK, extracellular signal regulated kinase; KLF4, kruppel-like factor 4; p-, phosphorylated; STAT3, signal transducers and activators of transcription; JAK, Janus kinase; p-, phosphorylated; VSMC, vascular smooth muscle cell.

PDGF-BB-induced STAT3 phosphorylation (Fig. 5A), ERK1/2 phosphorylation and increased KLF4 levels (Fig. 5B). Meanwhile, the results of CCK- 8 assay showed that blockade of JAK/STAT3 and ERK/KLF4 signaling pathways with AG490 or U0126 markedly attenuated PDGF-BB-induced VSMCs proliferation (Fig. 6A). Furthermore, the abnormal changes in phenotypic switching markers including $\alpha$-SMA, calponin and OPN were all rectified by AG490 or U0126 pretreatment in VSMCs response to PDGF-BB (Fig. 6B-E). These findings suggested that both JAK/STAT3 and ERK/KLF4 signaling pathways were required for the role of crocin in VSMCs.

\section{Discussion}

The present findings revealed some novel findings about crocin. Crocin effectively suppressed PDGF-BB-induced VSMCs proliferation. In addition, crocin prevented PDGF-BB-induced the VSMCs phenotype switch through inhibiting JAK/STAT3 and ERK/KLF4 signaling pathway.

Researches demonstrated that PDGF-BB plays a vital role in the formation of neointima (15). In normal mature vessels, the expression of PDGF and its receptors are very low or undetectable. In contrast, its expression is dramatically increased at vascular injury sites, followed by the increased activation of platelets and the recruitment of monocytes (16). The expression of exogenous PDGF in the arteries can induce intimal thickening through the stimulation of VSMCs proliferation and migration (17). PDGF promoted VSMCs to switch from the quiescent contractile phenotype to the synthetic phenotype by inhibiting expression of $\alpha$-SMA and calponin (18). Consistent with previous published studies, we found that PDGF-BB decreased $\alpha$-SMA and calponin expression but increased OPN expression. The following experiments found that crocin restored the expression of $\alpha$-SMA and calponin in a concentration dependent manner, accompanied by a decrease in cell proliferation. These data suggested that crocin halts the change toward a deleterious VSMCs phenotype induced by PDGF-BB. However, the mechanisms through which crocin inhibited PDGF-BB-induced VSMCs phenotypic switch remain unknown.

Janus kinase-signal transducer and activator of transcription (JAK-STAT) pathway, which is one of major downstream mediators of PDGF signaling, was activated in VSMCs exposed to PDGF-BB in our experiments. Previous studies identified that STAT3 signaling pathway is required for PDGF-BB stimulated VSMC proliferation (19). And suppressing JAK/STAT3 signaling pathway leading to the inhibition of proliferation and migration of smooth muscle cells (20). We revealed that crocin inhibited the activation of JAK/STAT3 pathway induced by PDGF-BB in a concentration dependent manner. Actually, Kim et al have demonstrated that crocin effectively suppressed constitutive STAT3 activation, translocation of STAT3 to the nucleus through induction of protein tyrosine phosphatase SHP-1 in multiple myeloma cells (21). Accordingly, we identified that suppression of STAT3 activation using $100 \mu \mathrm{M}$ crocin or a specific inhibitor resulted in VSMCs switching from the synthetic phenotype to the contractile phenotype again. The role of STAT3 in controlling 


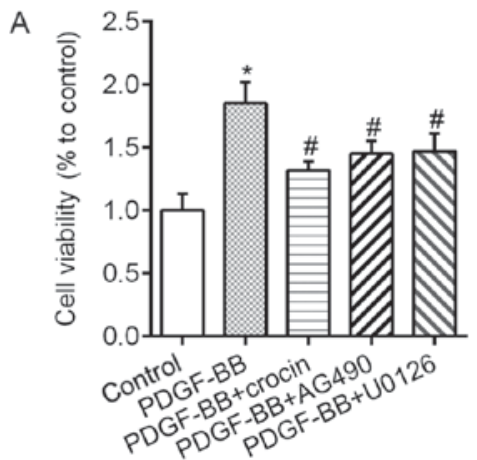

B
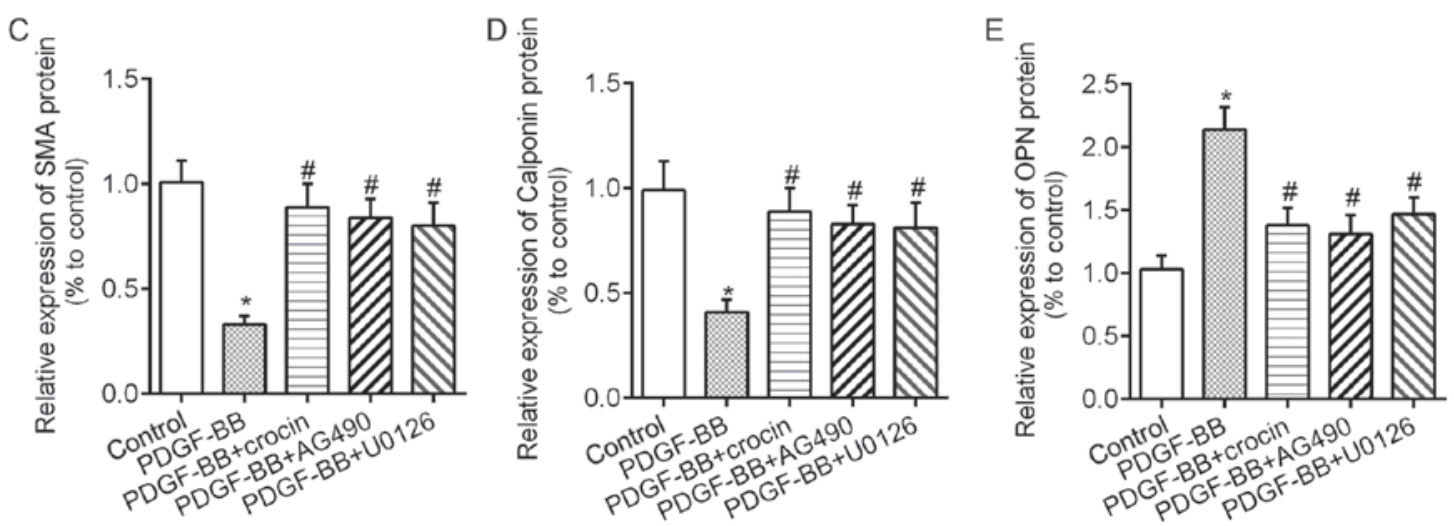

Figure 6. Crocin repressed PDGF-BB-induced VSMCs proliferation and phenotype switch via the JAK/signal transducers and activators of transcription 3 and ERK/kruppel-like factor 4 signaling pathways. (A) Cell Counting Kit-8 assay revealed cell proliferation in VSMCs treated with $20 \mathrm{ng} / \mathrm{ml}$ PDGF-BB, PDGF-BB + crocin $(100 \mu \mathrm{M})$, PDGF-BB + AG490 (10 $\mu \mathrm{M}$, a JAK inhibitor), PDGF-BB + U0126 (50 $\mu \mathrm{M}$, an ERK inhibitor). (B) Representative images showed the expression levels of $\alpha$-SMA, calponin and OPN protein. Bar graph showing the relative protein levels of (C) $\alpha$-SMA, (D) calponin and (E) OPN in the groups. "P<0.05 vs. control; " $\mathrm{P}<0.05$ vs. PDGF-BB. VSMC, vascular smooth muscle cell; PDGF-BB, platelet-derived growth factor-BB; ERK, extracellular signal regulated kinase; JAK, Janus kinase.

VSMCs phenotypic switch was supported by Liao et al study that activated STAT3 enhanced VSMCs proliferation and suppressed the expression of contractile proteins, whereas knockdown of endogenous STAT3 enhances VSMCs contractile phenotype (22). Although should be further validated in vivo, above data indicated that that JAK/STAT3 pathway was a potential therapeutic target for controlling phenotypic switch of VSMCs.

Besides activation of STAT transcription factors, PDGF-BB stimulation also leads to the initiation of the ERK signaling pathway $(23,24)$. Studies by several investigators showed that activation of ERK1/2 signaling contributes to promote VSMC proliferation $(25,26)$. KLF4 is the downstream target of ERK1/2 in the PDGF-BB-induced signaling pathway $(27,28)$. Consistent with another study (29), we noted that PDGF-BB induces phosphorylation of ERK1/2 and elevated KLF4 expression in VSMCs. Moreover, crocin treatment significantly inhibited PDGF-BB-induced activation of ERK/KLF4 signaling pathway. It is noteworthy that crocin can inhibit p-ERK1/2 in rat retina with ischemia/reperfusion injury (30) and improve lipid dysregulation in subacute diazinon exposure through inhibition of ERK1/2 activation in rat liver (31). We for the first time showed that crocin suppressed ERK1/2 pathway in cultured VSMCs. The role of ERK1/2 and KLF4 in VSMCs phenotypic switch had been well established (32-35). We also revealed that ERK1/2 inhibitor U0126 significantly reduced p-ERK1/2 and KLF4 levels. Consistent with crocin, U0126 reversed PDGF-BB-induced VSMCs phenotypic switch. Nevertheless, whether there excites another pathway that control KLF4 expression and the exact upstream kinases of ERK1/2 is still need to be identified. Collectively, our results suggested that inhibiting PDGF-BB-induced activation of ERK/KLF4 signaling pathway may have contributed to the inhibition of VSMC proliferation and phenotypic switch exerted by crocin. Of note, studies found JAK/ERK/STAT pathway was associated with cell proliferation, differentiation and survival $(36,37)$. The fact that JAK/ERK/STAT signaling pathway is also involved in attenuating cardiac ischemia/reperfusion injury (38) suggested an interaction between JAK and ERK signaling. Since PDGF-BB treatment influences other MAPK other MAPK pathways, such as JNK and p38 MAPK pathway (39), whether they participate in the inhibitory effects of crocin still needs to be defined.

Taken together, our results disclose for the first time that crocin inhibits PDGF-BB-induced VSMC phenotypic alteration and subsequent proliferation through regulating JAK/STAT3 and ERK/KLF4 signaling pathway. As VMSC proliferation is one of the key mechanisms involved in the development and progression of neointimal hyperplasia, which contributes to the pathogenesis of atherosclerosis and restenosis, use of crocin may be a potential way to restrain the progression of cardiovascular disease.

\section{Acknowledgements}

Not applicable. 


\section{Funding}

No funding was received.

\section{Availability of data and materials}

All data generated or analyzed during this study are included in this published article.

\section{Authors' contributions}

GQ designed the study and performed the statistical analysis; LT conducted all experiments and data correction; GQ and LT wrote the manuscript.

\section{Ethics approval and consent to participate}

The study was approved by the Institutional Animal Care and Use Committee of the First Hospital of China Medical University.

\section{Consent for publication}

Not applicable.

\section{Competing interests}

The authors declare that they have no competing interests.

\section{References}

1. Ding Y, Zhang M, Zhang W, Lu Q, Cai Z, Song P, Okon IS Xiao L and Zou MH: AMP-activated protein kinase alpha 2 deletion induces VSMC phenotypic switching and reduces features of atherosclerotic plaque stability. Circ Res 119: 718-730, 2016.

2. Yang F, Chen Q, He S, Yang M, Maguire EM, An W, Afzal TA, Luong LA, Zhang L and Xiao Q: miRNA-22 is a novel mediator of vascular smooth muscle cell phenotypic modulation and neointima formation. Circulation: pii: CIRCULATIONAHA.117.027799, 2017.

3. Yang X, Dong M, Wen H, Liu X, Zhang M, Ma L, Zhang C, Luan $\mathrm{X}, \mathrm{Lu} \mathrm{H}$ and Zhang Y: MiR-26a contributes to the PDGF-BB-induced phenotypic switch of vascular smooth muscle cells by suppressing Smad1. Oncotarget 8: 75844-75853, 2017.

4. Li G, Xie N, Yao Y, Zhang Y, Guo J, Feng Y, Lv F, Xiao RP and Cao CM: Identification of PI3K regulatory subunit $\mathrm{p} 55 \gamma$ as a novel inhibitor of vascular smooth muscle cell proliferation and neointimal formation. Cardiovasc Res 105: 75-85, 2015.

5. Kaimoto T, Yasuda O, Ohishi M, Mogi M, Takemura Y, Suhara T, Ogihara T, Fukuo K and Rakugi H: Nifedipine inhibits vascular smooth muscle cell dedifferentiation via downregulation of Akt signaling. Hypertension 56: 247-252, 2010.

6. Song IS, Jeong YJ, Park JH, Shim S and Jang SW: Chebulinic acid inhibits smooth muscle cell migration by suppressing PDGF-R $\beta$ phosphorylation and inhibiting matrix metalloproteinase-2 expression. Sci Rep 7: 11797, 2017.

7. Rahaiee S, Moini S, Hashemi M and Shojaosadati SA: Evaluation of antioxidant activities of bioactive compounds and various extracts obtained from saffron (Crocus sativus L.): A review. J Food Sci Technol 52: 1881-1888, 2015.

8. Amin A, Hamza AA, Daoud S, Khazanehdari K, Hrout AA, Baig B, Chaiboonchoe A, Adrian TE, Zaki N and SalehiAshtiani K: Saffron-based crocin prevents early lesions of liver cancer: In vivo, in vitro and network analyses. Recent Pat Anticancer Drug Discov 11: 121-133, 2016.

9. Mohammadzadeh L, Hosseinzadeh H, Abnous K and Razavi BM Neuroprotective potential of crocin against malathion-induced motor deficit and neurochemical alterations in rats. Environ Sci Pollut Res Int 25: 4904-4914, 2018.
10. He SY, Qian ZY, Tang FT, Wen N, Xu GL and Sheng L: Effect of crocin on experimental atherosclerosis in quails and its mechanisms. Life Sci 77: 907-921, 2005.

11. Li J, Lei HT, Cao L, Mi YN, Li S and Cao YX: Crocin alleviates coronary atherosclerosis via inhibiting lipid synthesis and inducing M2 macrophage polarization. Int Immunopharmacol 55: 120-127, 2018.

12. Chi J, Meng L, Pan S, Lin H, Zhai X, Liu L, Zhou C, Jiang C and Guo $\mathrm{H}$ : Primary culture of rat aortic vascular smooth muscle cells: A new method. Med Sci Monit 23: 4014-4020, 2017.

13. Liao Y, Hu X, Guo X, Zhang B, Xu W and Jiang H: Promoting effects of IL-23 on myocardial ischemia and reperfusion are associated with increased expression of IL-17A and upregulation of the JAK2-STAT3 signaling pathway. Mol Med Rep 16: 9309-9316, 2017.

14. Liu N, Xu L, Shi Y, Fang L, Gu H, Wang H, Ding X and Zhuang S: Pharmacologic targeting ERK1/2 attenuates the development and progression of hyperuricemic nephropathy in rats. Oncotarget 8: 33807-33826, 2017.

15. Wu B, Zhang L, Zhu YH, Zhang YE, Zheng F, Yang JY, Guo LY, Li XY, Wang L, Tang JM, et al: Mesoderm/mesenchyme homeobox gene 1 promotes vascular smooth muscle cell phenotypic modulation and vascular remodeling. Int J Cardiol 251: 82-89, 2018

16. Yan G, Wang Q, Hu S, Wang D, Qiao Y, Ma G, Tang C and Gu Y: Digoxin inhibits PDGF-BB-induced VSMC proliferation and migration through an increase in ILK signaling and attenuates neointima formation following carotid injury. Int J Mol Med 36: 1001-1011, 2015.

17. Park S, Kim JK, Oh CJ, Choi SH, Jeon JH and Lee IK: Scoparone interferes with STAT3-induced proliferation of vascular smooth muscle cells. Exp Mol Med 47: e145, 2015.

18. Salabei JK, Cummins TD, Singh M, Jones SP, Bhatnagar A and Hill BG: PDGF-mediated autophagy regulates vascular smooth muscle cell phenotype and resistance to oxidative stress. Biochem J 451: 375-388, 2013.

19. Simon AR, Takahashi S, Severgnini M, Fanburg BL and Cochran BH: Role of the JAK-STAT pathway in PDGF-stimulated proliferation of human airway smooth muscle cells. Am J Physiol Lung Cell Mol Physiol 282: L1296-L1304, 2002.

20. Ji Y, Yang X and Su H: Overexpression of microRNA-375 impedes platelet-derived growth factor-induced proliferation and migration of human fetal airway smooth muscle cells by targeting Janus kinase 2. Biomed Pharmacother 98: 69-75, 2018.

21. Kim B, Lee KY and Park B: Crocin suppresses constitutively active STAT3 through induction of protein tyrosine phosphatase SHP-1. J Cell Biochem 118: 3290-3298, 2017.

22. Liao XH, Wang N, Zhao DW, Zheng DL, Zheng L, Xing WJ, Ma WJ, Bao LY, Dong J and Zhang TC: STAT3 protein regulates vascular smooth muscle cell phenotypic switch by interaction with myocardin. J Biol Chem 290: 19641-19652, 2015.

23. Chang Y, Li JY, Jayakumar T, Hung SH, Lee WC, Manubolu M, Sheu JR and Hsu MJ: Ketamine, a clinically used anesthetic, inhibits vascular smooth muscle cell proliferation via PP2A-activated PI3K/Akt/ERK inhibition. Int J Mol Sci 18: pii: E2545, 2017.

24. Chen Q, Chen L, Kong D, Shao J, Wu L and Zheng S: Dihydroartemisinin alleviates bile duct ligation-induced liver fibrosis and hepatic stellate cell activation by interfering with the PDGF- $\beta R / E R K$ signaling pathway. Int Immunopharmacol 34: 250-258, 2016

25. Gharibi B, Ghuman MS and Hughes FJ: Akt- and Erk-mediated regulation of proliferation and differentiation during PDGFR $\beta$ induced MSC self-renewal. J Cell Mol Med 16: 2789-2801, 2012.

26. Kim JY, Kim KH, Lee WR, An HJ, Lee SJ, Han SM, Lee KG, Park YY, Kim KS, Lee YS, et al: Apamin inhibits PDGF-BBinduced vascular smooth muscle cell proliferation and migration through suppressions of activated Akt and Erk signaling pathway. Vascul Pharmacol 70: 8-14, 2015.

27. Kim MO, Kim SH, Cho YY, Nadas J, Jeong CH, Yao K, Kim DJ, Yu DH, Keum YS, Lee KY, et al: ERK1 and ERK2 regulate embryonic stem cell self-renewal through phosphorylation of Klf4. Nat Struct Mol Biol 19: 283-290, 2012.

28. Kawai-Kowase K and Owens GK: Multiple repressor pathways contribute to phenotypic switching of vascular smooth muscle cells. Am J Physiol Cell Physiol 292: C59-C69, 2007.

29. Chen S, Dong S, Li Z, Guo X, Zhang N, Yu B and Sun Y: Atorvastatin calcium inhibits PDGF- $\beta \beta$-induced proliferation and migration of VSMCs through the G0/G1 cell cycle arrest and suppression of activated PDGFR $\beta$-PI3K-Akt signaling cascade. Cell Physiol Biochem 44: 215-228, 2017. 
30. Chen L, Qi Y and Yang X: Neuroprotective effects of crocin against oxidative stress induced by ischemia/reperfusion injury in rat retina. Ophthalmic Res 54: 157-168, 2015.

31. Lari P, Rashedinia M, Abnous K and Hosseinzadeh H: Crocin improves lipid dysregulation in subacute diazinon exposure through ERK1/2 pathway in rat liver. Drug Res (Stuttg) 64: 301-305, 2014.

32. Wang TM, Chen KC, Hsu PY, Lin HF, Wang YS, Chen CY, Liao YC and Juo SH: microRNA let-7g suppresses PDGF-induced conversion of vascular smooth muscle cell into the synthetic phenotype. J Cell Mol Med 21: 3592-3601, 2017.

33. Sunaga H, Matsui H, Anjo S, Syamsunarno MR, Koitabashi N, Iso T, Matsuzaka T, Shimano H, Yokoyama T and Kurabayashi M: Elongation of long-chain fatty acid family member 6(Elovl6)-driven fatty acid metabolism regulates vascular smooth muscle cell phenotype through AMP-activated protein kinase/krüppel-like factor 4 (AMPK/KLF4) signaling. J Am Heart Assoc 5: pii: e004014, 2016.

34. Ouyang QF, Han Y,Lin ZH, Xie H, Xu CS and Xie LD: Fluvastatin upregulates the $\alpha 1 \mathrm{C}$ subunit of CaV1.2 channel expression in vascular smooth muscle cells via RhoA and ERK/p38 MAPK pathways. Dis Markers 2014: 237067, 2014.

35. Feng J, Ge S, Zhang L, Che $\mathrm{H}$ and Liang C: Aortic dissection is associated with reduced polycystin-1 expression, an abnormality that leads to increased ERK phosphorylation in vascular smooth muscle cells. Eur J Histochem 60: 2711, 2016.
36. Chang C, Zhao Y, Song G and She K: Resveratrol protects hippocampal neurons against cerebral ischemia-reperfusion injury via modulating JAK/ERK/STAT signaling pathway in rats. J Neuroimmunol 315: 9-14, 2018.

37. Ottani A, Giuliani D, Neri L, Calevro A, Canalini F, Vandini E, Cainazzo MM, Ruberto IA, Barbieri A, Rossi R and Guarini S: NDP-alpha-MSH attenuates heart and liver responses to myocardial reperfusion via the vagus nerve and JAK/ERK/STAT signaling. Eur J Pharmacol 769: 22-32, 2015.

38. Ottani A, Galantucci M, Ardimento E, Neri L, Canalini F, Calevro A,Zaffe D, Novellino E, Grieco P, Giuliani D and Guarini S: Modulation of the JAK/ERK/STAT signaling in melanocortininduced inhibition of local and systemic responses to myocardial ischemia/reperfusion. Pharmacol Res 72: 1-8, 2013.

39. Wilson JL, Yu J, Taylor L and Polgar P: Hyperplastic growth of pulmonary artery smooth muscle cells from subjects with pulmonary arterial hypertension is activated through JNK and p38 MAPK. PLoS One 10: e0123662, 2015.

This work is licensed under a Creative Commons Attribution-NonCommercial-NoDerivatives 4.0 International (CC BY-NC-ND 4.0) License. 